\title{
Animal-assisted therapy to promote ambulation in the hospital setting: Potentially effective but is it feasible?
}

\author{
Nancy L. Novotny ${ }^{* 1,2}$, Jackie Deibner ${ }^{3}$, Cheryl Herrmann ${ }^{3}$ \\ ${ }^{1}$ Mennonite College of Nursing, Illinois State University, United States \\ ${ }^{2}$ Methodist College, Peoria, IL, United States \\ ${ }^{3}$ UnityPoint Health-Methodist, Peoria, IL, United States
}

Received: January 31, 2015

DOI: $10.5430 /$ jnep.v5n $7 \mathrm{p} 123$
Accepted: April 19, 2015

Online Published: May 11, 2015

URL: http://dx.doi.org/10.5430/jnep.v5n7p123

\begin{abstract}
Objective: Preliminary studies demonstrating efficacy of the use of animal assisted therapy (AAT) to promote ambulation in the hospital setting support the need for additional studies using rigorous designs to determine its potential for use in the clinical setting. To determine the feasibility of a randomized controlled trial of AAT in the hospital setting to promote ambulation in cardiac patients the objectives were to examine recruitment yields; acceptability of therapy; and identification of safety, patient care, resource, and methodological challenges in using AAT with inpatients from multiple perspectives.

Methods: This mixed methods study used structured interviews with patients who declined participation in AAT, participants who walked with a therapy dog, nursing personnel, and primary care providers. Participants' clinical and demographic data were abstracted from healthcare records. Descriptive statistics were reported for quantitative data and narrative responses were analyzed using content analysis.

Results: Unit personnel and participants perceived AAT to be acceptable, without negatively impacting unit resources, and safe - even among overweight or patients at higher risk of falling. Many patients for whom this intervention would be appropriate declined participation. Availability of therapy dogs was inconsistent and required alteration of plans for AAT on several days.

Conclusions: Major obstacles to routine use of hospital-based AAT need to be addressed when designing a study to compare the effectiveness of AAT with other interventions. A sufficient pool of therapy dogs and their handlers would need to be in place to facilitate its use in a randomized controlled trial to promote ambulation in clinical practice.
\end{abstract}

Key Words: Animal assisted therapy, Cardiovascular nursing, Ambulation, Feasibility, Hospitalized adults

\section{INTRODUCTION}

Animal assisted therapy (AAT) is a potentially useful complementary, goal-directed therapeutic intervention to positively impact patients' physical and psychosocial functioning across healthcare settings. ${ }^{[1,2]}$ Several small studies demonstrated desirable physiological, psychological, and behavioral effects of AAT in patients hospitalized with cardiovascular problems. ${ }^{[3,4]}$ Therapeutic benefits of AAT are the- oretically attributed to decreased catecholamine, increased endorphins, and neuronal activity. ${ }^{[5]}$

Exercise is beneficial in a variety of cardiac conditions. ${ }^{[6-9]}$ Complications of immobility are well known. Evidence supporting the importance of ambulation during cardiac-related hospitalizations is substantial, albeit primarily a low level. ${ }^{[10]}$ In spite of its relative safety and benefits, active promotion of ambulation during hospitalization remains inadequate and is

*Correspondence: Nancy L. Novotny; Email: nlnovot@ilstu.edu; Address: Mennonite College of Nursing, Illinois State University, United States. 
associated with nurses' perceived importance and existence of protocols. ${ }^{[11]}$ Ambulation was the most frequently missed type of nursing care in a survey of 4086 nurses across ten hospitals, with $33 \%$ of nurses identifying ambulation as frequently or always missed. ${ }^{[12]}$ These findings were confirmed in the time and motion study of 47 older patients in an acute care setting in which no mobility events occurred in $32 \%$ of the patients and nurses did not initiate any walks within the room or hallways for the subset of dependent patients. ${ }^{[13]}$

Use of AAT in promotion of ambulation has been studied in hospitalized patients with heart failure. ${ }^{[3,4]}$ Willingness to walk and distance walked were significantly greater with AAT participants in comparison to the historical controls. ${ }^{[3]}$ Selection, baseline dissimilarities, and evolution of clinical practices may have contributed to the observed group differences identified in the pilot studies. Further, studies of the efficacy of AAT in this population have been limited to single AAT exposures. ${ }^{[3,4]}$

Duration of effects derived after a single AAT intervention is unknown. In clinical practice, meaningful effectiveness of AAT may require frequent sessions. Although AAT is a potentially useful strategy to promote ambulation in this population, the practicality of its routine use in the clinical setting and establishment of an integrated, hospital-based AAT program capable of producing sustained effects have not been documented. Further, it remains unclear whether the use of other motivators would produce results as effective as AAT. The types of interventions which are the most effective, motivating, practical, and least resource-intensive to mobilize hospitalized adults is not yet known. Marino concluded from his review of two meta-analyses and other quantitative studies of AAT that the available evidence is not sufficiently rigorous to provide support for the idea that an animal, opposed to other novel motivator, is needed for the therapeutic effects attributed to AAT to date. ${ }^{[14]}$ For these reasons, well-designed randomized controlled studies are needed.

Additionally, reports of ill effects of AAT on patients due to phobias, exposure to zoonotic infections, allergies, bites, or scratches in the healthcare setting are rare; however, safety concerns across all those involved in AAT within the inpatient setting have not been fully described in the literature. Several studies that compared infection rates or zoonotic infections post implementation of an AAT program have not identified cause for concern, however, veterinarians caution overreliance on these findings due to insufficient information about surveillance methods, methodological quality, and whether the most potentially harmful pathogens commonly identified in the hospital environment were monitored. ${ }^{[15]}$
To minimize the risks to humans and pet therapy dogs in the hospital setting, it is important to institute appropriate precautions and adhere to guidelines for AAT. ${ }^{[15,16]}$ This may include regular canine health screenings, the obligation of hospital staff to ensure adherence to infection control procedures, and compliance with AAT-related policies in the hospital setting.

\section{Objectives and research questions}

Based on the evidence currently available, two questionswill AAT work and can AAT work in the hospital setting to promote ambulation in cardiac patients - have yet to be answered. The objective of a feasibility study is to determine whether it will be practical to conduct a randomized controlled trial of a specific intervention in a specific setting but not to examine effectiveness of the intervention. ${ }^{[17]}$ A feasibility study of use of AAT within the hospital setting was warranted prior to initiating a randomized controlled trial comparing AAT with non-AAT motivators to promote ambulation throughout hospitalization in cardiac patients. Feasibility studies of health promotion interventions, such as promotion of ambulation, may focus upon the acceptability of the intervention by target subjects and other stakeholders as well as the implementation, practicality, and integration of an intervention. ${ }^{[18]}$

This feasibility study involved consideration of AAT from multiple perspectives to explore challenges including willingness to participate, acceptability of the intervention, difficulties with implementation of AAT, impact upon patient care and available resources within a nursing unit, and safety issues.

These important considerations were addressed in this study by answering three research questions: (1) What percent of hospitalized cardiovascular patients would participate in AAT? (2) Does AAT in an inpatient setting impact patient safety, patient care, or use of resources? (3) What are the perceptions of involved patients, nurses (RN), certified nursing assistants (CNA), and primary care providers (PCP) about use of therapy animals to promote ambulation?

\section{Methods}

A descriptive feasibility study was conducted at one Midwestern medical center over 5 months. After Institutional Review Board approval, investigators screened health care records of adult inpatients on one progressive cardiac unit once a week. Eligible patients had a cardiovascular diagnosis, ambulated safely within the past day, were capable of participating in an interview in English, did not have a cognitive deficit or dog allergy, and were not on isolation precautions. Patients could only participate once. Willing pa- 
tients provided written informed consent. Patients unwilling to participate were asked to share their reasons.

Policies at the medical center concerning use of AAT specified limitations and requirements in order to avoid patient problems. All AAT visits needed to be supervised, the animal was to be appropriated restrained, and the animal was never to be left alone with a patient. Also, a copy of an agreement had to be filed which indicated the dog handler ensured the cleanliness and health of the animal, appropriate behavior, and proof of current immunizations.

Investigators abstracted demographic and clinical information from participants' health care records, including weight, height, gait, use of ambulatory devices, and fall risk based on a modified Morse Fall Scale. Weight status, using CDC categories, was based on the calculated body mass index.

Because the focus of this study was designed to measure feasibility, not the effectiveness of ambulation, participants were walked with certified therapy dogs only once. The CNA and/or RN helped the patient prepare for the walk and assisted the patient during the walk as needed. The dog walked at the patient's side while the handler maintained control of the animal without assisting in the patient's care. The handler and patient each held one end of a double leash. A record was kept of any fall, accident, or safety issue during the intervention.

Investigators conducted structured interviews to elicit perceptions about AAT after each walk with participants and with their nurse or CNA when only the CNA assisted with the walk. Participants, RNs, and CNAs were also asked if they had a dog. Nurses and CNAs were asked about equipment used, number of staff who assisted during the walk, safety concerns, the need for any modifications of care for the participant or other patients on the unit during AAT, and whether it was more time consuming to ambulate the patient with the therapy dog. Some questions provided pre-defined response options or a Likert-type scale, while others allowed for open-ended responses. After the last participant was enrolled, primary care providers of patients on the unit were interviewed about their views of the use of therapy animals on the unit. These providers included hospitalists, family practice residents, and cardiologists.

The investigators reviewed the narrative responses to openended items that were provided by patients, RN/CNAs, and primary care providers. Using content analysis, these responses were coded and then organized into similar categories to identify common themes. Quantitative analyses were performed using SPSS Version 21.0. ${ }^{[19]}$ No data was missing for patient interviews. A few responses for RN/CNA interviews were missing and are identified in the results. Responses to interview items and the patients' demographic and clinical variables measured at the interval level were described using means and standard deviations or medians and ranges for non-normal distributions. Frequencies and percentages were reported for categorical variables. Associations between participant and RN/CNA perceptions were examined using Spearman correlation coefficients.

\section{RESUlts}

\section{Research question 1}

On the days screening occurred, 35\% (90/256) of patients on the unit were eligible and 84 of these were asked to participate. Four patients were not asked because the therapy animal did not arrive and two patients were off the unit at the time. Among those approached, 55\% (46/84) agreed to enroll.

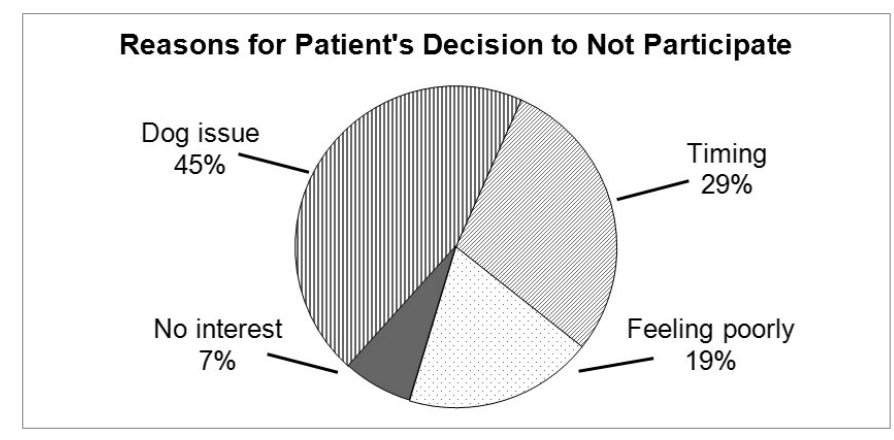

Figure 1. Reasons patients decided not to participate in the study $(n=31)$

Of the 38 non-participants, $82 \%$ shared reasons for declining. As shown in Figure 1, the reasons fit into four major themes. The most frequent reasons for refusal related specifically to dogs. Comments exemplifying this category included: "me and dogs don't get along", "I'm afraid dog will get away from me", and "want to walk only with a person". The times that dogs were available to walk influenced decisions of patients who were waiting for a scheduled procedure or preparing 
to go home. Some patients did not feel well enough, citing weakness, nausea or difficulty breathing. A general lack of interest was indicated by comments such as "just don't want to" or "I can walk (without a dog)".

Of the consenting patients, $80 \%$ (37/46) actually participated in the AAT walk. On the designated AAT days, one to seven participants walked with a dog. Interferences to participation involved patients who were off the unit or receiving education $(n=4)$, dogs not arriving $(n=3)$, patient feeling lightheaded $(\mathrm{n}=1)$, and patient who indicated the doctor told her not to walk with the dog due to balance issues $(n=1)$.

Table 1. Participating patient characteristics $(\mathrm{N}=37)$

\begin{tabular}{ll}
\hline Characteristics & $\mathbf{n}(\%)$ \\
\hline Sex & $16(43)$ \\
Female & $21(57)$ \\
Male & \\
Primary cardiac condition & $9(24)$ \\
Arrhythmias & $11(30)$ \\
Heart failure & $6(16)$ \\
Myocardial infarction, chest pain, or acute coronary syndrome & $4(11)$ \\
Post-cardiac surgery & $7(19)$ \\
Vascular diseases & \\
Fall risk category & $1(3)$ \\
Low & $22(59)$ \\
Moderate & $14(38)$ \\
High & \\
Self-assessed health status & $4(11)$ \\
Excellent or very good & $27(73)$ \\
Good & $6(16)$ \\
Fair or poor & \\
Weight category per body mass index & $2(5)$ \\
Underweight $(<18.5)$ & $5(14)$ \\
Normal $(18.5-24.9)$ & $12(32)$ \\
Overweight $(25-29.9)$ & $18(49)$ \\
Obese $(\geq 30)$ &
\end{tabular}

Characteristics of the participants who walked with a dog are summarized in Table 1. Participants ranged from 26 to 89 years of age with a mean of $70(\mathrm{SD}=15)$ years and slightly over half were male. Weights ranged from 101 to 282 and the average was $191(\mathrm{SD}=48)$ pounds. Body mass index ranged from 17.9 to 54.6 with a mean of $30(\mathrm{SD}=8)$; nearly half of the participants were obese. The self-rated health status of most participants was rated as good to excellent. Heart failure was the most frequent primary cardiac problem.

Nearly all participants were classified as having a moderate or high risk of falling. The median fall risk score was 45 and scores ranged from 20 to 100. Participants' gaits were normal $(52 \%)$, weak $(43 \%)$, or impaired $(5 \%)$. A walker was used for ambulatory assistance by $19 \%$ (7/37) of participants.

Structured interviews were conducted with 13 RNs and 24 CNAs. Fewer patients (35\%) than RN/CNAs (57\%) had a dog of their own. Personnel involved in the AAT activity included one CNA during $62 \%$ of the walks, both an RN and CNA during $24 \%$, one RN during $11 \%$, and no personnel during $3 \%$.

\section{Research question 2}

The potential impact of AAT on patients first began with examination of safety risks to patients and staff. No falls, accidents, or other safety-related incidents occurred during the AAT. No patient commented upon any safety issue. Three safety concerns reported by the RN/CNAs included one patient becoming wobbly which was unrelated to the dog or handler, one dog who "was all over" prompting concern that the patient would trip on the dog's leash, and one instance where the patient "kept bending over to kiss and touch the dog".

Second, there was no perceived negative impact upon patient care. The RN/CNAs denied the need for any modifications of care with any participants or other patients on the unit (responses available for 36/37 RN/CNA interviews) due to the AAT. Third, equipment and nursing personnel's time involved with AAT were considered. Assistive equipment was used during $44 \%$ of the walks: Oxygen tanks $6 \%$, IV pole $26 \%$, gait belt $9 \%$, walkers $81 \%$, and one prosthesis. No canes or carts were used, although a wheelchair was used four times in lieu of a walker. For two patients the RN/CNAs reported it took one to two minutes longer for dog introductions and petting.

\section{Research question 3}

Stakeholder perceptions about AAT to promote ambulation included those of the patients, RN/CNAs, and primary care providers. Patients offered positive feedback about the experience: $95 \%$ reported they would be willing to walk with a dog in the hospital every day and $97 \%$ believed that therapy dogs should be used. All patients responded favorably to the question, "Did you enjoy walking with the dog?" Over $90 \%$ (34/37) of patients reported complete enjoyment during the walk and the remaining three enjoyed it somewhat or a lot. In response to the question, "Did the visit from the dog effect how you felt?" 59\% (22/37) felt much better, 30\% (11/37) felt somewhat better, and $11 \%$ (4/37) said it had no influence on how they felt. Distance was recorded for 35 of the 37 AAT walks. Walks averaged 310 feet $(S D=140)$ feet and ranged from 10 to 700 feet.

Table 2 summarizes responses to questions which were posed to both participants and nursing personnel concerning whether AAT influenced the patient's willingness to walk or the distance walked. Perceptions about its influence were overall positive among patients and nursing personnel. 
Table 2. Patient and nursing personnel perceptions about AAT

\begin{tabular}{|c|c|c|}
\hline \multirow{2}{*}{ Perceptions } & Patient & RN/CNA \\
\hline & n (\%) & n (\%) \\
\hline \multicolumn{3}{|l|}{ AAT influence on taking a walk ${ }^{*}$} \\
\hline Not at all & $4(11)$ & $2(6)$ \\
\hline Somewhat or a lot & $4(11)$ & $4(11)$ \\
\hline Strongly & $29(78)$ & $30(83)$ \\
\hline \multicolumn{3}{|l|}{ AAT influence on distance walked* } \\
\hline Strongly or somewhat discouraged & 0 & 0 \\
\hline No influence & $10(27)$ & $3(8)$ \\
\hline Somewhat encouraged & $10(27)$ & $7(19)$ \\
\hline Strongly encouraged & $17(46)$ & $26(72)$ \\
\hline \multicolumn{3}{|l|}{ Themes from comments ${ }^{* *}$} \\
\hline Promoted sense of well-being & $8(28)$ & $17(42)$ \\
\hline Promoted ambulation & $3(11)$ & $4(10)$ \\
\hline Dog specific & $10(36)$ & $6(15)$ \\
\hline Positive perceptions about AAT program & $7(25)$ & $13(33)$ \\
\hline
\end{tabular}

${ }^{*}$ One response missing from RN/CNA caring for the participant who did not need nursing assistance during walk; ${ }^{* *}$ A comment could relate to multiple themes; percentages do not total $100 \%$.

Several patient responses were positively associated. "Did the dog make you want to take a walk?" was associated with "How much did the animal influence how far you walked?" $(r=.372, p=.023)$, "Did you enjoy walking with the dog?" $(r=.381, p=.020)$, and "Did the visit from the dog effect how you felt?" $(r=.389, p=.017)$. Participant feelings after the walk with the dog were also associated with the amount of enjoyment ( $r=.370, p=.024)$ and how far the patient perceived the distance walked $(r=.465, p=.004)$.

Associations between RN/CNA perceptions and other variables were also examined. There was no association between the patient's and RN/CNA's perceptions of the AAT influence upon the patient's desire or willingness to walk ( $r=.146$, $p=.507)$ or upon the distance walked $(r=.198, p=.253)$. There was an association between the RN/CNAs perception of influence of AAT on how far the patient walked with the recorded distance walked $(r=.398, p=.020)$. Lastly, none of the participants' or RN/CNAs perceptions were associated with either having one's own dog or the participant's health status or gait.

Investigators identified four major themes within the comments that 19 participants and 24 RN/CNAs shared after walks: Encouragement of ambulation, promotion of a sense of well-being, dog-specific issues, and positive perceptions about the AAT program. The frequencies of comments concerning these themes within each stakeholder group are also summarized in Table 2. Comments such as "good, that patient did 300 feet", "he usually doesn't like to walk", and "If I didn't want to walk, a dog would make me walk." are indicative of those found in the promoted ambulation cate- gory. Promotion of a sense of well-being was suggested by statements such as "it was a good thing for him, he was depressed, and this made him smile". Examples of dog-specific comments included "patient really liked her" (the dog) and "dog was clean". Lastly, "patients always seem to love the dog program" was typical of comments which were included in the AAT program category.

Six of the 9 primary care providers interviewed were aware that AAT was being used on the unit to promote ambulation. All agreed that AAT was a positive activity. Comments included "I think there should be more dogs" and "I think they are one of the best things we do here; they should be mandatory." One respondent acknowledged that all patients do not like animals.

\section{Discussion}

This study examined factors which may influence implementation of regular integration of AAT to promote ambulation within an acute care setting. Because participation in this study involved minimal respondent burden, the nonparticipation rate is a valuable indicator of how inpatients may welcome involvement in AAT. Only $41 \%$ of eligible patients actually participated in AAT. Many patients who may have benefited from AAT did not participate. The rate of enrollment in a randomized controlled trial would be expected to be much lower and prolong the time required to conduct such a study.

These results provide a better understanding of reasons to decline participation in AAT. Fear or dislike of dogs and interference with other hospital activities were patients' most 
frequent reasons for non-participation. Identification of alternate forms of motivation is needed for patients who dislike the essence of AAT.

All patients who chose involvement in AAT acknowledged enjoyment in the activity. Further, $78 \%$ reported it strongly influenced them to take a walk. These results affirm participants' acceptability of AAT and willingness to be involved among volunteers.

The overall positive feedback by professionals and staff concerning AAT was consistent with previously reported healthcare personnel attitudes after implementation of a dog visitation program for hospitalized pediatric patients. ${ }^{[20]}$ Across both these studies, positive staff and patient attitudes were common themes, with overall acceptance of dog-therapy and recognition of the associated relief from the humdrum hospital environment. Staff supported this study and demonstrated notable excitement on "dog days". After several weeks nursing personnel began alerting the investigators to potentially eligible patients.

Complementary therapies must accommodate priority treatments and institutional schedules yet not drain available institutional resources, or cause undue harm. Potential negative impact of AAT upon patient safety, patient care, or use of resources was explored in multiple ways. There was a concern that use of AAT for ambulation may create a "parade" in the hallways with the patient, dog handler, dog, nursing personnel, and equipment (IV pole, oxygen tank, walker, wheelchair), thereby posing safety risks to staff, visitors or other patients. Hallways at this institution, however, were wide enough that others could walk around the parade. This study did not compare resource utilization between ambulation with and without AAT yet there was no obvious increase in equipment used during AAT. With infrequent minimal additional time required, there was no apparent drain on resources.

No adverse events occurred, however the sample was small. The dogs remained well-behaved, on the double leash, and under the handler's control at all times. Rare concerns related to fall risks and patients' receptivity to dogs were identified, yet no problems or near misses were encountered. Risks were minimized by the dog walking alongside versus ahead of patients and using a short leash. Of interest, most patients were overweight or obese, yet there was no increased safety risk in this group.

In this study the timing of arrival, consistently at 10 am worked well. By then rounds, assessments, and morning treatments were complete. Pre-identification of appropriate participants allowed the nursing team time to have patients ready to ambulate.

To successfully study AAT as a goal directed intervention in a randomized controlled trial, a sufficient pool of AAT dogs and handlers who are available most days of the week at a designated time is essential. Although the data collection for this study occurred only on one day per week, the dog-handler teams were unexpectedly unavailable during 8 of the 20 weeks due to illness, vacations, birthing puppies, and stormy weather that frightened the dogs. This created issues with patients looking forward to AAT only to be told the "dogs aren't coming". Identification of this barrier in this feasibility study is of potentially great importance when planning a randomized controlled clinical trial. In a future trial, the inability to adhere to administration of planned treatments at the intervals planned may cause underestimation of treatment effects and thereby may contribute to a potential for Type II errors. ${ }^{[9,21]}$

There are several national therapy dog certification organizations and available data suggest 10000 to 20000 handleranimal teams may be registered with a single organization, although these include teams that are located outside of the United States. ${ }^{[22,23]}$ The geographical distribution of teams may not coincide with desirable locations for clinical trials. In this Midwestern community only seven teams were available at the time this study was conducted. Of these, only three teams were willing to walk with patients. Intermittent availability of the animals and handlers, who typically volunteer services, is not an uncommon situation. ${ }^{[5]}$ Further, repeated engagement with a multiple strangers or the unpredictability involved in the hospital environment may increase stress, harm therapy dogs physiologically, and limit the number of consecutive days a dog-handler team may be available. ${ }^{[24,25]}$ These challenges could severely restrict enrollment of sufficient subjects, increase study costs, or decrease treatment fidelity or treatment adherence within a randomized controlled clinical trial. Clear communication with the potential pool of dog handlers about the availability of the team and willingness to walk with patients would be critical to help ensure the ability to consistently administer the AAT intervention during a patient's hospitalization.

\section{Conclusions}

AAT is feasible on a busy, progressive cardiac unit. It caused no patient safety issues and did not contribute to a meaningful increase in staff time. In addition, it was well received and supported by all healthcare providers. These findings also support the idea that AAT is a motivator for those who readily opt to engage in this therapy. Feasibility of its use to promote ambulation in acutely ill hospitalized cardiac 
patients, however, may be compromised by limited acceptability among the majority of appropriate patient candidates, having sufficient dog-handler teams willing to participate for daily use, and difficulties involved in matching the schedules of volunteer pet therapy handlers and inpatients. Further, length of stay in progressive cardiac units has been decreasing over the years and opportunities to motivate patients to begin early ambulation need to be initiated as soon as possible. For this reason, exploration of continued use of AAT in outpatient cardiac rehabilitation programs is another area worthy of investigation.

This study provides useful information to plan for randomized controlled studies of AAT in the hospital setting. Investi- gators should consider reasons patients may not readily seek or accept involvement in AAT therapies in designing patient enrollment strategies. An important potential barrier to the success of ensuring fidelity of treatment interventions within a randomized control trial is availability of sufficient doghandler teams. The sustained effects of AAT on ambulation in hospitalized cardiac patients compared to other feasible strategies that clinicians may use to motivate participation in early ambulation merit further exploration.

\section{CONFlicts OF InTEREST Disclosure}

The authors declare that there is no conflict of interest statement.

\section{REFERENCES}

[1] Snipelisky D, Burton MC. Canine-assisted therapy in the inpatient setting. South Med J. 2014; 107: 265-273. PMid:24937523 http://dx.doi.org/10.1097/SMJ.0000000000000090

[2] Kamioka H, Okada S, Tsutani K, et al. Effectiveness of animalassisted therapy: A systematic review of randomized controlled trials. Complement Ther Med. 2014; 22: 371-390. PMid:24731910 http://dx.doi.org/10.1016/j.ctim.2013.12.016

[3] Abate SV, Zucconi M, Boxer BA. Impact of canine-assisted ambulation on hospitalized chronic heart failure patients' ambulation outcomes and satisfaction: A pilot study. J Cardiovasc Nurs. 2011; 26: 224-230. PMid:21263346 http://dx.doi.org/10.1097/J CN. 0b013e3182010bd6

[4] Cole KM, Gawlinski A, Steers N, et al. Animal-assisted therapy in patients hospitalized with heart failure. Am J Crit Care. 2007; 16: 575-588. PMid:17962502

[5] Marcus DA. The science behind animal-assisted therapy. Curr Pain Headache Rep. 2013; 17: 322321-3217:322.

[6] Davies EJ, Moxham T, Rees K, et al. Exercise training for systolic heart failure: Cochrane systematic review and meta-analysis. Eur J Heart Fail. 2010; 12: 706-715. PMid:20494922 http://dx.doi.o $\mathrm{rg} / 10.1093 /$ eurjhf/hfq056

[7] Giacomantonio NB, Bredin SS, Foulds HJ, et al. A systematic review of the health benefits of exercise rehabilitation in persons living with atrial fibrillation. Can J Cardiol. 2013; 9: 483-491. PMid:23200094 http://dx.doi.org/10.1016/j.cjca.2012.07.003

[8] Heran BS, Chen JM, Ebrahim S, et al. Exercise-based cardiac rehabilitation for coronary heart disease (Review). Cochrane Database Syst Rev. 2011; 6. http://dx.doi.org/10.1002/14651858.cd 001800 .pub2

[9] Lawler PR, Filion KB, Eisenberg MJ. Efficacy of exercise-based cardiac rehabilitation post-myocardial infarction: A systematic review and meta-analysis of randomized controlled trials. Am Heart J. 2011; 162: 571-584. PMid:21982647 http://dx.doi.org/10.1016/j .ahj.2011.07.017

[10] Pron G. Early mobilization and ambulation in hospitalized heart failure patients: A rapid review. Toronto, ON., Health Quality Ontario; 2013. Available from: http: //www.hqontario.ca/Portals/0/Documents/eds/rapid-r eviews/early-mobilization-ambulation-130121-en.pdf

Published by Sciedu Press
[11] Winkelman C, Peereboom K. Staff perceived barriers and facilitators. Crit Care Nurse. 2010; 30: S13-S16. PMid:20360441 http: //dx.doi.org/10.4037/ccn2010393

[12] Kalisch BJ, Tschannen D, Lee H, et al. Hospital variation in missed nursing care. Am J Med Qual. 2011; 26: 291-299. PMid:21642601 http://dx.doi.org/10.1177/1062860610395929

[13] Doherty-King B, Yoon JY, Pecanac K, et al. Frequency and duration of nursing care related to older patient mobility. Image J Nurs Sch. 2014; 46: 20-17. PMid:24112775 http://dx.doi .org/10.1111 /jnu. 12047

[14] Marino L. Construct validity of animal assisted therapy and activities: How important is the animal in AAT? Anthrozoös. 2012; 25: S139-151. http://dx.doi.org/10.2752/175303712X13353430377219

[15] Lefebvre SL, Peregrine AS, Golab GC, et al. A veterinary perspective on the recently published guidelines for animal-assisted interventions in health-care facilities. JAVMA. 2008; 233: 394 402. PMid:18673025 http://dx.doi.org/10.2460/javma. 233 .3 .394

[16] Murthy R, Bearman G, Brown S, et al. Animals in healthcare facilities: recommendations to minimize potential risks. Infect Control \& Hosp Epidemiol. 2015; 36: 495-516. http://dx.doi.org/10.10 $17 /$ ice.2015.15

[17] Abbott JH. Randomized clinical trials (RCTs) and preliminary feasibility and pilot studies: What they are and are not. J Orthop Sports Phys Ther. 2014; 44: 555-558. PMid:25082389 http://dx.doi.o rg/10.2519/jospt.2014.0110

[18] Bowen DJ, Kreuter M, Spring B, et al. How we design feasibility studies. Am J Prevent Med. 2009; 36: 452-257. PMid:19362699 http://dx.doi.org/10.1016/j.amepre. 2009.02.002

[19] IBM Corporation. IBM SPSS Statistics for Windows, 2012.Version 21.0. Armonk, NY: IBM Corp.

[20] Moody WJ, King R, O'Rourke S. Attitudes of paediatric medical ward staff to a dog visitation programme. J Clin Nurs. 2002; 11: 537-544. http://dx.doi.org/10.1046/j.1365-2702.20 $02.00618 . x$

[21] Persch AC, Page SJ. Protocol development, treatment fidelity, adherence to treatment, and quality control. Am J Occup Ther. 2013; 67: 146-153. PMid:23433268 http://dx.doi.org/10.5014/a jot. 2013.006213 
[22] American Kennel Club: List of therapy dog training organizations [Internet]. C2015 [cited 2015 Jan 31]. Available from: http: //www.akc.org/akctherapydog/organizations.cfm

[23] Therapy Dog Information: Local Therapy Animal Organizations [Internet]. C2015 [cited 2015 Jan 31]. Available from: http://www . therapydoginfo.net/organizations.html

[24] Haubenhofer DK, Kirchengast S. Physiological arousal for com- panion dogs working with their owners in animal-assisted activities and animal-assisted therapy. J Appl Anim Welf Sci. 2006; 9: 165172. PMid:16956319 http://dx.doi.org/10.1207/s15327604 jaws0902_5

[25] Ng Z, Pierce BJ, Otto CM. The effect of dog-human interaction on cortisol and behavior in registered animal-assisted activity dogs. Appl Anim Behav Sci. 2014; 159: 69-81. http://dx.doi.org/10.10 $16 / j$.applanim.2014.07.009 